\title{
Accidental Decannulation Following Placement of a Tracheostomy Tube
}

\author{
Alexander C White MD, Elaine Purcell MD, Mary Beth Urquhart RN MBA, \\ Bernard Joseph MD, and Heidi H O'Connor MD
}

\begin{abstract}
BACKGROUND: Accidental decannulation is a cause of substantial morbidity and mortality in patients in long-term acute care hospitals who require a tracheostomy tube. OBJECTIVE: To analyze features of accidental decannulation (AD) following placement of a tracheostomy tube, and to implement strategies to reduce the problem. METHODS: An analysis of data collected prospectively for quality management in a long-term acute care hospital was performed. RESULTS: AD occurred at a rate of $4.2 \pm 0.9 / 1,000$ tracheostomy days over a 7 month period. Factors associated with AD included mental status changes, increased secretions, and change of shift. Following the implementation of a series of interventions (staff education on risk factors for AD and best tracheostomy care practice; increased availability of telemetry and oximetry; and signage to identify patients at high risk of $A D$ ), the incidence of $A D$ over a subsequent 7 month period was significantly reduced, to $2.7 \pm 1.9 / 1,000$ tracheostomy days. In addition the numbers of multiple, unmonitored, unreported, and night shift ADs were all significantly reduced. CONCLUSIONS: Targeted interventions can significantly reduce both the incidence of $\mathrm{AD}$ following tracheostomy and associated morbidity. Best practice guidelines to help minimize AD in patients with tracheostomy tubes are proposed. Key words: accidental decannulation; morbidity; mortality; long-term care; tracheostomy; tracheotomy; interventions; quality. [Respir Care 2012;57(12):2019-2025. ( 2012 Daedalus Enterprises]
\end{abstract}

\section{Introduction}

The loss of an artificial airway may result in serious consequences. Unlike accidental extubation following translaryngeal intubation. ${ }^{1-4}$ accidental decannulation (AD) is not a well recognized complication of tracheostomy in adults, especially in the post-acute setting, and most reports are single cases. ${ }^{5-8}$ More data are available on $\mathrm{AD}$ occurring in the pediatric population, either in the hospital ${ }^{9,10}$ or home setting. ${ }^{11}$

The authors are affiliated with the Department of Pulmonary and Sleep Medicine, Rose Kalman Research Center, New England Sinai Hospital, Stoughton, Massachusetts, with the exception of Dr Purcell, who is affiliated with the Pulmonary, Critical Care, and Sleep Division, Tufts Medical Center, Boston, Massachusetts, and Ms Urquhart, who is affiliated with the Department of Quality Management, New England Sinai Hospital, Stoughton, Massachusetts.

The study was partly supported by unrestricted grants from the Rose Kalman Research Center at New England Sinai Hospital. The authors have disclosed no conflicts of interest.
The number of patients receiving prolonged mechanical ventilation via tracheostomy has been increasing. ${ }^{12} \mathrm{~A}$ tracheostomy tube secures the airway, reduces the risk of laryngeal injury, ${ }^{13}$ may enhance weaning, ${ }^{14-16}$ and facilitates the transfer of the patient from an ICU to a less acute setting such as a respiratory step down unit or a long-term acute care hospital (LTACH). ${ }^{17}$

This LTACH based study, prompted by 2 sentinel events, was performed in order to determine the incidence of $\mathrm{AD}$, to identify factors related to $\mathrm{AD}$, to design and implement strategies to reduce $\mathrm{AD}$, and to measure the impact of these strategies on $\mathrm{AD}$ incidence.

\footnotetext{
Correspondence: Alexander C White MD, Department of Pulmonary and Sleep Medicine, Rose Kalman Research Center, New England Sinai Hospital, 150 York Street, Stoughton MA 02072. E-mail: awhite@ nesinai.org.
}

DOI: $10.4187 /$ respcare. 01627 


\section{Methods}

\section{Setting}

The study was performed at an LTACH system (3 sites) located in Massachusetts. The LTACH has 160 active beds and receives an average of 2,500 admissions per year. Approximately $35 \%$ of all admitted patients $(n=56)$ have a tracheostomy tube in position, 65\% of whom require prolonged mechanical ventilation. The remaining patients have a tracheostomy tube for airway protection. An increasing number of patients are admitted for management of noninvasive mechanical ventilation. Comprehensive medical and rehabilitative care is delivered at the LTACH system to patients with complex medical diseases, with an average stay of 25 days. There is focus on weaning from mechanical ventilation, noninvasive mechanical ventilation, management of multiple comorbidities, and specialized wound care. Physical, occupational, and speech therapy, along with nutritional support, are all available.

See the Related Editorial on Page 2133

Routine tracheostomy care, including tracheostomy tube changes, is provided by trained respiratory therapists, supervised by pulmonary/critical care physicians. Staffing is allocated on a unit basis (on average 20 beds), with one respiratory therapist allocated per unit. There is a nurse to patient ratio 1:5, with nursing aides also assisting with patient care. Videobronchoscopy is available for managing complex airways.

A waiver for informed consent was obtained from the institutional review board at New England Sinai Hospital.

\section{Definition of Accidental Decannulation}

$\mathrm{AD}$ was defined as the unexpected removal of a tracheostomy tube, either by the patient or during care delivery (such as turning patient). Scheduled decannulations were excluded.

\section{Data Collection}

Following 2 sentinel $\mathrm{AD}$ events resulting in death, a prospective data collection system was initiated on January 1,2007 , by the quality management department, to determine the frequency of $\mathrm{AD}$ and the factor(s) associated with each reported AD. Incident reports filed by staff were the primary data source for $95 \%$ of AD events, and the remaining events were identified from staff meetings, review of medical records, and billing compliance. Once an $\mathrm{AD}$ event was identified, a chart review was performed

\section{QUICK LOOK}

\section{Current knowledge}

Accidental decannulation is a cause of substantial morbidity and mortality in patients in long-term acute care hospitals who require a tracheostomy tube. Techniques to reduce the incidence of accidental decannulation are not well described.

\section{What this paper contributes to our knowledge}

Accidental decannulation occurs in 4.2 $\pm 0.9 / 1,000$ tracheostomy days. Factors associated with accidental decannulation include mental status changes, increased secretions, and change of shift. Implementation of a series of interventions can reduce the incidence of accidental decannulation.

and, if necessary, staff interviews performed to determine timing of the event, possible associated factors, and complications following the AD. Data were collected on paper and then entered into a spread sheet (Excel, Microsoft, Redmond, Washington).

\section{Time Frame of Data Collection}

The 7 month period from January 1, 2007, through July 31, 2007, was considered a "baseline period," as it predated the implementation of any formal interventions to reduce $\mathrm{AD}$. Following an analysis of factors associated with $\mathrm{AD}$ reported during the baseline period (root cause analysis), interventions to reduce $\mathrm{AD}$ incidence were developed by a multidisciplinary committee representing medicine, nursing, and respiratory therapy. The interventions were designed to address deficits in care of the patient with a tracheostomy in the following areas: caregiver knowledge, process of care, and equipment. The interventions were implemented over 3 month period from $\mathrm{Au}$ gust 1, 2007, through October 31, 2007. Follow-up AD data were then collected over a subsequent 7 month period (November 1, 2007, through May 2008). All AD event data collected over each 7 month period are expressed as mean $\pm \mathrm{SD}$.

\section{Statistical Analysis}

Continuous variables were analyzed using the Wilcoxon 2-sample test. Statistical significance was assumed when $P<.05$. 


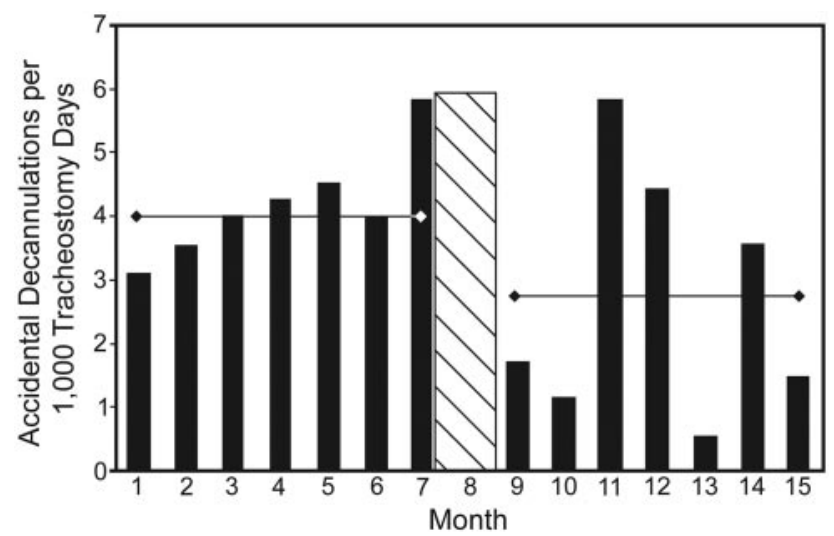

Figure. Bar chart showing significant reduction in average accidental decannulation rate. Months 1-7 are prior to interventions (hatched area). Months $9-15$ are post interventions. ${ }^{*} P=.04$, compared with average accidental decannulation rate prior to interventions.

\section{Results}

\section{Accidental Decannulation Rate}

The $\mathrm{AD}$ rate/1,000 tracheostomy days per month is shown in the Figure. The timing and effect of the interventions are shown. The AD rate during the 7 month period prior to the intervention was $4.2 \pm 0.9 / 1,000$ tracheostomy days, occurring in 60 patients. Other data are shown on Table 1. Thirty-two percent of ADs occurred in patients undergoing prolonged mechanical ventilation via tracheostomy. In $12 \%$ of ADs a sleep apnea tube was in place. Following $\mathrm{AD}, \mathrm{S}_{\mathrm{pO}_{2}}$ was maintained above $95 \%$ in $88 \%$ of patients and fell to $<90 \%$ in $12 \%$ of patients. Stoma closure occurred in $9 \%(n=3)$ of patients following AD. No deaths from AD occurred during the study period or during the subsequent 2 years.

\section{Factors Associated With Accidental Decannulation}

A number of factors (patient or system related) were identified as being associated with AD (Table 2).

\section{Interventions to Prevent Accidental Decannulation}

Interventions were developed and then implemented with a focus on increasing caregiver knowledge and enhancing equipment. The goal of the educational component was to correct identified knowledge deficits in tracheostomy care. Mandatory educational modules (Table 3) were presented to medical, nursing, and respiratory therapy staff, either in a classroom or at the bedside during the 3 month intervention period. Following a review of baseline AD data, additional equipment needs were identified (see Table 3).
Revisions were also made to policies to clarify expectations for monitoring patients at risk of AD.

\section{The Effect of the Interventions on Accidental Decannulation}

Data obtained in the 7 month follow-up period following the interventions (see Table 1) demonstrated a significant reduction in the $\mathrm{AD}$ rate, to $2.7 \pm 1.9 / 1,000$ tracheostomy days ( $P=.04$ ) in a total of 33 patients. There was a significant reduction in episodes of desaturation following $\mathrm{AD}$ following the interventions (pre-intervention $0.6 \pm 0.8$ events, compared with post intervention $0.4 \pm 0.5$ events, $P=.02$ ). There was a significant reduction in the number of ADs occurring in patients not monitored with oximetry (pre-intervention $2.6 \pm 0.9$, compared with $0.9 \pm 1.1, P=.004)$. In addition there was a significant reduction in the number of patients with multiple AD events, the number of AD events in patients on mechanical ventilation, and in AD events occurring during the night shift following the interventions. Other data are shown in Table 1 .

\section{Discussion}

In this study we have identified factors associated with $\mathrm{AD}$ and have demonstrated a reduction in the $\mathrm{AD}$ rate following the implementation of a series of interventions. This study was motivated by 2 AD related deaths. The first occurred following $\mathrm{AD}$ in a patient with a recently placed tracheostomy tube, during routine skin care. The tracheostomy tube was inadvertently replaced in a false passage in the anterior mediastinum, a previously reported complication of AD. ${ }^{18}$ The second death occurred following an AD that remained undetected for a critical period of time, due to equipment failure.

The clinical implications of any AD depend on a number of factors, including the indications for tracheostomy tube placement and the skill level of the responding staff. $\mathrm{AD}$ occurring in a patient with a mature tracheostomy stoma (ie, tracheostomy tube in position for at least $7 \mathrm{~d}$ ) and with normal neck anatomy should be a benign event. $\mathrm{AD}$ occurring in this setting is uncomplicated because the tracheostomy tube can usually be easily reinserted. In fact, the decision to permanently decannulate the patient may be informed by an AD that was uncomplicated.

However, AD may result in important morbidity and even mortality when it occurs in patients with a recently placed $(<7 \mathrm{~d})$ tracheostomy tube. Those patients with substantial airway pathology, increased neck circumference, marginal oxygenation, or in whom the tracheostomy tube is essential for mechanical ventilation appear to be particularly vulnerable. In these situations, airway patency 


\section{Accidental Decannulation Following Placement of a Tracheostomy Tube}

Table 1. Comparison of Accidental Decannulation Events Before and After Interventions

\begin{tabular}{|c|c|c|c|}
\hline & Pre & Post & $P$ \\
\hline Time period, months & 7 & 7 & \\
\hline Number of patients with AD events & 60 & 33 & \\
\hline BMI, $\mathrm{kg} / \mathrm{m}^{2}$ & $32 \pm 11$ & $28 \pm 7$ & .14 \\
\hline Rate, ADs/1,000 tracheostomy days & $4.2 \pm 0.9$ & $2.7 \pm 1.9$ & .04 \\
\hline Single AD event & $3.9 \pm 2$ & $2 \pm 1.7$ & .05 \\
\hline Multiple AD events & $4.7 \pm 1.8$ & $2.7 \pm 1.7$ & .03 \\
\hline 2nd $\mathrm{AD}$ event, patient unmonitored via telemetry or oximetry & $2.9 \pm 1.6$ & $1.3 \pm 1.4$ & .04 \\
\hline Patient on mechanical ventilation & $2.0 \pm 1.5$ & $0.3 \pm 0.5$ & .008 \\
\hline $\mathrm{AD}$ event during change of shift & $1.6 \pm 1.3$ & $0.9 \pm 0.7$ & .10 \\
\hline \multicolumn{4}{|l|}{ Shift } \\
\hline 7:00 АM-3:00 РМ & $2.6 \pm 1.6$ & $1.4 \pm 1.3$ & .08 \\
\hline 3:00 PM-11:00 PM & $2.9 \pm 1.3$ & $2.4 \pm 2.1$ & .33 \\
\hline 11:00 PM-7:00 Ам & $3.1 \pm 1.3$ & $0.9 \pm 0.7$ & $<.001$ \\
\hline Discovered during rounds & $0.14 \pm 0.4$ & $0.3 \pm 0.5$ & .02 \\
\hline Sleep apnea tube & $1.3 \pm 1.7$ & $0 \pm 0$ & .03 \\
\hline No incident report filed & $2 \pm 1.2$ & $0 \pm 0$ & .003 \\
\hline Followed by stoma closure & $0.4 \pm 0.5$ & $0 \pm 0$ & .03 \\
\hline $\begin{array}{l}\mathrm{AD}=\text { accidental decannulation } \\
\mathrm{BMI}=\text { body mass index }\end{array}$ & & & \\
\hline
\end{tabular}

Table 2. Factors Associated With Accidental Decannulation

\begin{tabular}{lc}
\hline \hline & no. (\%) \\
\hline Patient-Related & \\
$\quad$ Mental status change & $42(43)$ \\
Traumatic brain injury & $5(5)$ \\
Increased airway secretions & $13(13.5)$ \\
Recent tracheostomy change. & $4(4.25)$ \\
Vigorous coughing & $1(1)$ \\
Tracheostomy placed $<7$ days & $1(1)$ \\
Within 24 hours of admission to long-term & $1(1)$ \\
$\quad$ acute care hospital & \\
System-Related & $13(13.5)$ \\
Change of shift & $13(13.5)$ \\
No limb restraints in confused patient & $4(4.25)$ \\
Tracheostomy ties loose &
\end{tabular}

must be quickly re-established in order to avoid prolonged hypoxemia, organ failure, or death.

In this study we identified a number of factors associated with $\mathrm{AD}$. In greater than $40 \%$ of $\mathrm{AD}$ events, mental status changes such as agitation and delirium appeared to be important. Although delirium is a well described longterm complication of critical illness, ${ }^{19}$ it has not been well studied in the LTACH setting. The recognition and treatment of delirium, along with sedation management, are important when liberating patients from mechanical ventilation. It is necessary to optimize respiratory drive $\mathrm{e}^{20-22}$ and enhance patient participation in rehabilitative efforts, while at the same time avoiding mental status changes that could result in AD.
Table 3. Interventions Implemented to Reduce Both the Incidence of Accidental Decannulation and Morbidity Following Accidental Decannulation

\footnotetext{
Educational Components for Staff

Identification of patients considered at increased risk for $\mathrm{AD}$

Education on the use of "stay sutures"

Recent tracheostomy: risks

Review of an updated monitoring policy for patients at risk of $\mathrm{AD}$

Review of the need for more than one caregiver to be present during turning/moving patient with tracheostomy tube in position

Mandatory hourly rounds on all patients

Introduction of mandatory reporting of all $\mathrm{AD}$ events

Awareness of alarm fatigue

Equipment

Replacement tracheostomy tube at bedside

Labeled syringe to deflate cuff at bedside

Sign at head of bed identifying type of tracheostomy tube in position and date of last change

Mini-tracheostomy kit available in all code carts

Expanded availability of telemetry and pulse oximetry

$\mathrm{AD}=$ accidental decannulation
}

In $14 \%$ of cases of $\mathrm{AD}$, increased pulmonary secretions were reported to be present. ${ }^{23}$ Typical strategies include effectively treating pulmonary infections, optimizing cardiac function, and maintaining adequate oral hygiene. Anticholinergic medications to reduce secretions can also be helpful ${ }^{24}$ but may affect mental status. More work is needed to determine if secretions play a role in $\mathrm{AD}$. 
Fourteen percent of all ADs occurred during change of shift. Hand-off communication from one shift to the next is essential for patient safety, but direct patient supervision can be compromised as a result. Patient supervision can be maintained during hand-off, by using either a "walking report" format or a hand-off located at the bedside. Either approach may reduce accidental falls and improve calllight response. ${ }^{25,26} \mathrm{We}$ have recently found bedside handoffs for both nursing and respiratory therapy to be very effective in reducing $\mathrm{AD}$ in high risk patients in the LTACH setting.

In $14 \%$ of $\mathrm{ADs}$, limb restraints were not implemented despite the presence of mental status changes. The use of limb restraints should be minimized in the hospital setting, to both reduce the likelihood of trauma or injury to the restrained limb as well as to preserve patient autonomy and dignity. ${ }^{27}$ However, minimizing restraint use in patients with medical devices always carries a risk of deliberate or accidental device removal. In a recent survey designed to identify caregiver perceptions of risk factors for unplanned extubation in the ICU, $72 \%$ of caregivers considered the absence of physical restraints a risk factor for accidental extubation. ${ }^{28}$ It is not clear from our data whether the lack of restraints per se increased the risk of AD. The use of sitters in the room, rather than restraints, to prevent device removal is effective, but can be costly. High dependence units within the LTACH with greater supervision and a higher nursing/patient ratio for high risk patients may be needed for some patients.

The tracheostomy tube must be secured with ties to prevent $\mathrm{AD}$. Excessive tightening of ties must be avoided to prevent hospital acquired pressure ulcer formation around the neck. Surgeons usually suture the flange of the tracheostomy tube to the skin as an additional safety measure to prevent AD. ${ }^{29}$ Stay sutures may also be placed to facilitate re-insertion of a tracheostomy tube following AD. ${ }^{30} \mathrm{~Pa}-$ tients at risk of $\mathrm{AD}$ need to be identified at the time of tracheotomy to ensure the device is appropriately secured prior to discharging the patient to a less acute care setting.

During the observational period we identified deficits in knowledge regarding care of the patient with a tracheostomy. Like other LTACHs, the hospital had experienced a substantial increase in patient acuity. ${ }^{31}$ Staff were caring for patients who were discharged from ICUs with higher levels of acuity, including substantial numbers with recently placed tracheostomy tubes in position.

Following the introduction of targeted interventions designed to correct deficiencies identified in the observational period, we demonstrated a significant reduction in $\mathrm{AD}$ rate in the follow-up time period. Others have demonstrated reductions in unplanned extubations following endotracheal intubation using similar strategies. ${ }^{23}$ In addition, the number of multiple $\mathrm{AD}$ events, along with $\mathrm{ADs}$ occurring in unmonitored patients and those on mechani- cal ventilation, were all significantly reduced. There was a significant reduction in $\mathrm{AD}$ occurring during the night shift, but not during the day and evening shifts. We suspect this reflects better tracheostomy care in general. The significant reduction in the numbers of ADs occurring without an incident report being filed reflects staff being encouraged to report adverse events, thus helping provide data for quality improvement. The availability of telemetry and bedside oximetry was greatly expanded with state of the art equipment being installed. Patients with tracheostomy tubes were admitted only to units with this enhanced monitoring capability. Monitoring guidelines were implemented to ensure that appropriate patients were monitored and staff were educated about monitoring fatigue. A significant reduction in unmonitored $\mathrm{AD}$ events was noted following these interventions. The results suggest that the interventions had a significant impact on both caregiver knowledge and process of care. In the obese population, carefully selecting the size of the tracheostomy tube based on predictors of trachea to skin thickness may also help reduce AD. ${ }^{32}$

More recently we have been able to further reduce $\mathrm{AD}$ to negligible levels by mandating hourly rounding by respiratory therapists on all patients with a tracheostomy and changing work flow patterns to ensure that this is practical. Other strategies used to improve quality, such as mistakeproofing and force function, could also be adapted to tracheostomy care to try and eliminate AD. ${ }^{33}$

The AD rate in the LTACH setting could be a useful benchmark of quality, in much the same way as central line infections has become a benchmark for rating acute care hospitals on delivery of care. It is imperative that LTACHs have strategies to optimize the safety of patients admitted with tracheostomy tubes. High risk patients must be identified so that strategies to avoid $\mathrm{AD}$ during the initial 10 days (when patients are most vulnerable) can be implemented. Unless the receiving LTACH has caregivers trained in safely changing tracheostomy tubes, along with necessary equipment to manage the change and any unexpected complications (such as airway loss), the initial tracheostomy tube change should be performed at the acute care hospital. This could be done either prior to discharge to the LTACH or arranged in the out-patient setting. LTACH staff must be aware that attempts by unskilled practitioners to reinsert a freshly placed tracheostomy tube that has become dislodged can result in a misplaced tube with subsequent loss of the airway, which in turn may be fatal.

This study has some limitations. Some benign AD events with no consequences may have never been identified, and as a result the $\mathrm{AD}$ rate may have been underestimated. Reliable differentiation between staff-initiated and patientinitiated $\mathrm{AD}$ was not possible. This study was based at a 


\section{Accidental Decannulation Following Placement of a Tracheostomy Tube}

single LTACH system, and results may not be applicable to other LTACHs.

\section{Conclusions}

In conclusion, the rate of $\mathrm{AD}$ in patients with tracheostomies in an LTACH was significantly reduced following interventions designed to improve both caregiver knowledge and the care delivery processes. We have developed best practice recommendations (below) to help minimize $\mathrm{AD}$ in the LTACH setting.

\section{Best Practice Recommendations}

Knowledge: Optimize caregiver (physician, mid-level providers, nursing, respiratory therapy) knowledge about AD. Relevant topics include upper airway anatomy, tracheostomy (indications, types, early and late complications), decannulation (planned and accidental), and risks factors for AD.

Safe practice: Develop care pathways to reduce risks of $\mathrm{AD}$ during routine care. Examples include: reduce risk of $\mathrm{AD}$ during turning and cleaning of patients at high risk of complications following AD (one additional staff member responsible for monitoring the position of the tracheostomy tube during care); ensure optimal tracheostomy tie tension to avoid both AD and tie related hospital acquired pressure ulcer formation; time out procedure prior to any tracheostomy tube change (correct patient, correct replacement tracheostomy tube, correct monitoring available, contingency planning in event of failure to replace tube); and hourly rounding by nursing and respiratory therapists to check on tracheostomy tie tension and tracheostomy tube position.

Identify and monitor the high risk patient: difficult tube placement, difficult endotracheal intubation, mental status changes/delirium recognition, tracheostomy tube in place $<7$ days. Clearly identify tracheostomy type, size, length, date of last change in the medical record.

Optimize available monitoring capabilities and equipment: telemetry, tracheoscopy, aids to intubation (eg, GlideScope), adequate tracheostomy tube size and length, spare tracheostomy tube at bedside.

Develop strong collaborations with staff with expertise in complex airway management (eg, otolaryngology and interventional pulmonology).

\section{ACKNOWLEDGMENTS}

We thank John Devlin PharmD for his invaluable constructive criticism of the manuscript.

\section{REFERENCES}

1. Mion LC, Minnick AF, Leipzig R, Catrambone CD, Johnson ME. Patient-initiated device removal in intensive care units: a national prevalence study. Crit Care Med 2007;35(12):2714-2720.
2. de Lassence A, Alberti C, Azoulay E, Le Miere E, Cheval C, Vincent $\mathrm{F}$, et al. Impact of unplanned extubation and reintubation after weaning on nosocomial pneumonia risk in the intensive care unit: a prospective multicenter study. Anesthesiology 2002;97(1):148-156.

3. Epstein SK, Nevins ML, Chung J. Effect of unplanned extubation on outcome of mechanical ventilation. Am J Respir Crit Care Med 2000;161(6): 1912-1916.

4. Pesiri AJ. Two-year study of the prevention of unintentional extubation. Crit Care Nurs Q 1994;17(3):35-39.

5. Kapadia FN, Bajan KB, Raje KV. Airway accidents in intubated intensive care unit patients: an epidemiological study. Crit Care Med 2000;28(3):659-664.

6. De Leyn P, Bedert L, Delcroix M, Depuydt P, Lauwers G, Sokolov $\mathrm{Y}$, et al. Tracheotomy: clinical review and guidelines. Eur J Cardiothorac Surg 2007;32(3):412-421.

7. Seay SJ, Gay SL, Strauss M. Tracheostomy emergencies: correcting accidental decannulation or displaced tracheostomy tube. Am J Nurs 2002;102(3):59-63.

8. Rauf K, Zwaal JW. Accidental decannulation after surgical tracheostomy. Anaesthesia 2004;59(5):517.

9. Colman KL, Mandell DL, Simons JP. Impact of stoma maturation on pediatric tracheostomy-related complications. Arch Otolaryngol Head Neck Surg 2010;136(5):471-474.

10. Black TL, Fernandes ET, Carr MG. Preventing accidental decannulations following tracheostomy. J Pediatr Surg 1988;23(2):143.

11. Tibballs J, Henning R, Robertson CF, Massie J, Hochmann M, Carter $\mathrm{B}$, et al. A home respiratory support programme for children by parents and layperson carers. J Paediatr Child Health 2010;46(1-2): $57-62$.

12. Cox CE, Carson SS, Holmes GM, Howard A, Carey TS. Increase in tracheostomy for prolonged mechanical ventilation in North Carolina, 1993-2002. Crit Care Med 2004;32(11):2219-2226.

13. Loh KS, Irish JC. Traumatic complications of intubation and other airway management procedures. Anesthesiol Clin N Am 2002;20(4): 953-969.

14. Davis K Jr, Campbell RS, Johannigman JA, Valente JF, Branson RD. Changes in respiratory mechanics after tracheostomy. Arch Surg 1999;134(1):59-62.

15. Diehl JL, El Atrous S, Touchard D, Lemaire F, Brochard L. Changes in the work of breathing induced by tracheotomy in ventilator-dependent patients. Am J Respir Crit Care Med 1999;159(2):383-388.

16. Quinnell TG, Pilsworth S, Shneerson JM, Smith IE. Prolonged invasive ventilation following acute ventilatory failure in COPD: weaning results, survival, and the role of noninvasive ventilation. Chest 2006;129(1):133-139.

17. White AC, O'Connor HH, Kirby K. Prolonged mechanical ventilation: review of care settings and an update on professional reimbursement. Chest 2008;133(2):539-545.

18. O'Connor HH, White AC. Tracheostomy decannulation. Respir Care 2010;55(8):1076-1081.

19. Desai SV, Law TJ, Needham DM. Long-term complications of critical care. Crit Care Med 2011;39(2):371-379.

20. Girard TD, Kress JP, Fuchs BD, Thomason JW, Schweickert WD, Pun BT, et al. Efficacy and safety of a paired sedation and ventilator weaning protocol for mechanically ventilated patients in intensive care (Awakening and Breathing Controlled Trial): a randomised controlled trial. Lancet 2008;371(9607):126-134.

21. Schweickert WD, Gehlbach BK, Pohlman AS, Hall JB, Kress JP. Daily interruption of sedative infusions and complications of critical illness in mechanically ventilated patients. Crit Care Med 2004; 32(6): 1272-1276.

22. King MS, Render ML, Ely EW, Watson PL. Liberation and animation: strategies to minimize brain dysfunction in critically ill patients. Semin Respir Crit Care Med 2010;31(1):87-96. 


\section{Accidental Decannulation Following Placement of a Tracheostomy Tube}

23. Lucas da Silva PS, de Carvalho WB. Unplanned extubation in pediatric critically ill patients: a systematic review and best practice recommendations. Pediatr Crit Care Med 2010;11(2):287-294.

24. Tassinari D, Poggi B, Fantini M, Tamburini E, Sartori S. Treating sialorrhea with transdermal scopolamine. Exploiting a side effect to treat an uncommon symptom in cancer patients. Support Care Cancer 2005;13(7):559-561.

25. Spanke MT, Thomas T. Nursing assistant walking report at change of shift. J Nurs Care Qual 2010;25(3):261-265.

26. Griffin T. Bringing change-of-shift report to the bedside: a patientand family-centered approach. J Perinat Neonatal Nurs 2010;24(4): 348-353.

27. Center for Medicare and Medicaid Services. State Operations Manual. Appendix A - survey protocol, regulations and interpretive guidelines for hospitals. http://www.cms.gov/manuals/Downloads/ som107ap_a hospitals.pdf Accessed September 21, 2012.
28. Tanios MA, Epstein SK, Livelo J, Teres D. Can we identify patients at high risk for unplanned extubation? A large-scale multidisciplinary survey. Respir Care 2010;55(5):561-568.

29. Liew L, Gibbins N, Oyarzabal M. How I do it: securing tracheostomy tubes. Eur Arch Otorhinolaryngol 2008;265(5):607-608.

30. Burke A. The advantages of stay sutures with tracheostomy. Ann R Coll Surg Engl 1981;63(6):426-428.

31. Carson SS, Cox CE, Holmes GM, Howard A, Carey TS. The changing epidemiology of mechanical ventilation: a population-based study. J Intensive Care Med 2006;21(3):173-182.

32. Szeto C, Kost K, Hanley JA, Roy A, Christou N. A simple method to predict pretracheal tissue thickness to prevent accidental decannulation in the obese. Otolaryngol Head Neck Surg 2010;143(2): 223-229.

33. van Beuzekom M, Boer F, Akerboom S, Hudson P. Patient safety: latent risk factors. Br J Anaesth 2010;105(1):52-59.

This article is approved for Continuing Respiratory Care Education credit. For information and to obtain your CRCE

(free to AARC members) visit www.rcjournal.com

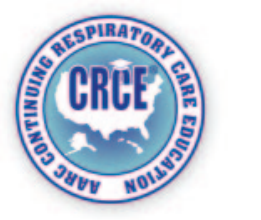

